\title{
CONTINUOUS EVOLUTION IN COLABORATIONS BETWEEN MOLDOVAN AND ITALIAN CHEMISTS
}

Bilateral collaborations represent an efficient tool for fruitful valorization by scientific communities of their own research potential and proved beneficial in most research areas. This is true indeed, since the most valuable research results lay on the interface of different research areas, and developing collaborations between researches working in complementary fields is most likely to provide valuable solutions.

In the past decades Moldovan researches have been constrained to develop such collaborations with different international partners due to the precarious state of the Modovan research infrastructure. Institute of Chemistry was in the forefront of this strategy, with multiple examples of joint research projects developed first of all with European colleagues. One of such examples is provided by the Laboratory of Terpenoid Chemistry, led by Prof. Nicon Ungur which involves Italian partners from Centro Nazionale della Ricerca (CNR), namely Institute of Biomolecular Chemistry (ICB), Naples. This collaboration has started in 1994, with a kind agreement of Prof. Guido Cimino, at that time director of ICB, to provide hosting to Prof. Nicon Ungur in order to develop a research project devoted to synthesis of marine natural products. This start was extremely successful and the collaboration got a tremendous development. The last year provided additional opportunities in this context, connected to the agreement between ASM and CNR to develop joint bilateral projects. This circumstance has let the Moldovan chemists to host the Italian partners in Chisinau and to organize in premiere for the last 20 years an international seminar devoted to the chemistry of natural products.

The formal reason of this seminar was a bilateral ASM-CNR project aimed to the investigation of terpenes from cannabinoid family. The Cannabis sativa plant represents a isolation source for more then 400 chemical compounds, of which more then 100 compounds possess prenylated aromatic structures. These mero-terpenoids have been called phytocannabinoids and are found almost exclusively in this plant. The structural feature of these compounds is given by the presence of two distinct structural parts of differnt biogenetical origine: the aromatic part, specifically a derivative of chromene and the isoprenoidic part. Biosynthetically, the initial steps involve condensation of the aromatic part (olivetol) with geranyl pyrophosphate to provide the first, most simple representative of the family - cannabigerol (scheme 1). Following enzymatic cyclisations lead to different families of phytocannabinoids, including the most known cannabichromene- and cannabidiol-type compounds.
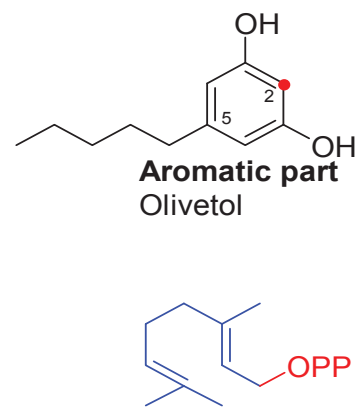

Terpenic part

Geranyl pyrophosphate
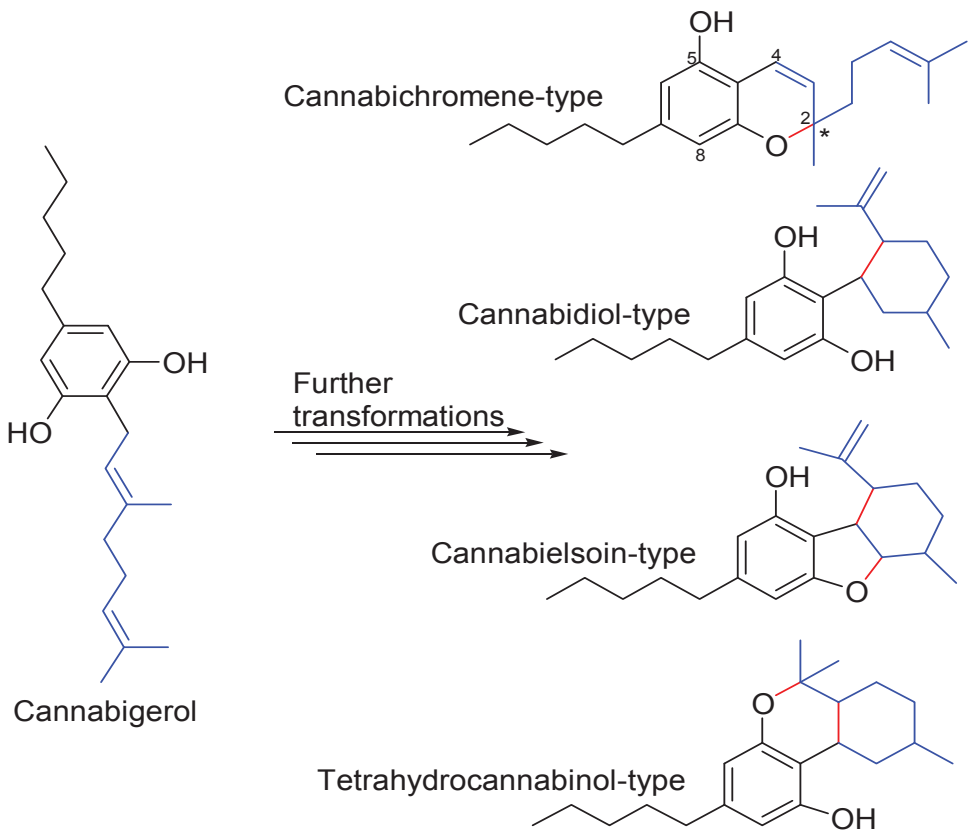

Scheme 1

For evident reasons, the basic attention during the study of this natural product family has been paid to $\Delta$ 9-tetrahydrocannabinol ( $\triangle 9$-THC), which is the well known compound with psychotropic activity from cannabis. Nevertheless, recent studies has shown that other non- psychotropic phytocannabinoids possess a broad spectrum of biological activities, most of them being associated with therapeutic applications. Among these, cannabichromenetype compounds have drawn attention due to their antiinflamatory, analgesic, antimicrobial, antiproliferative and bone growing stimulation actions. It made them challenging targets for chemical synthesis. 
Our current ASM-CNR project includes elaboration of novel methods for the chemical synthesis, evaluation of absolute stereochemistry and biological activity of cannabichromene-type compounds. The synthetic aproach is considered, provided the fact that the most relevant compounds of this class are still not avilable from natural sources in sufficient ammounts and consequently can not be explored for therapeutical purpose. In the same time, determination of the absolute stereochemistry of most representatives is not a trivial task and can be sucsesfully solved only basing on a complex aproach, including concurent synthesis and degradation studies. Finally, broadening the structural features of prenylated cannabinoids, especially on varying the structure of isoprenic residue can lead to novel substances with new and still unexplored properties.

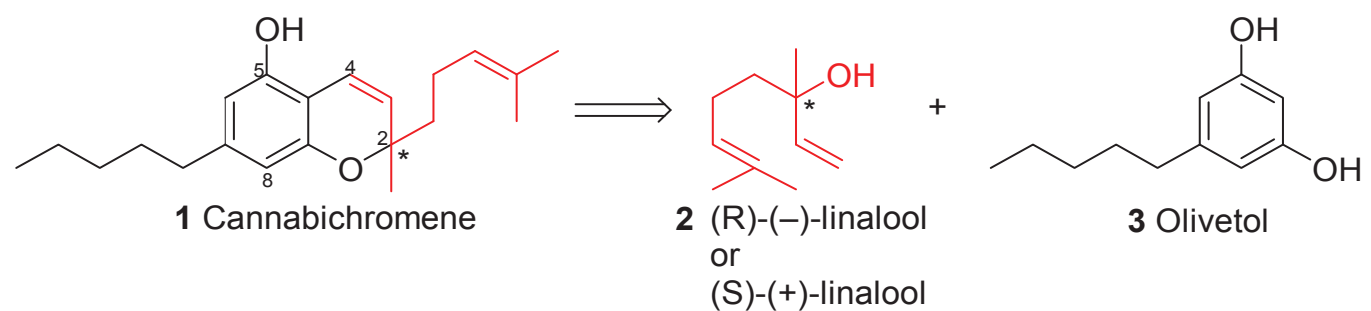

Scheme 2

Basing on available preliminary information, we have focused our project on the synthesis of cannabichromene one of the basic representatives of prenylated cannabinoids, having as the final goal the elaboration of a general procedure for the synthesis of a whole series of prenylated cannabinoids. We intend to apply a combination of enantioselective and diastereoselective synthesis in order to access desired prenylated chromenes in enantiomericaly pure form (scheme 2). Following degradation studies and stereochemical correlation will allow determination of chromene absolute stereochemistry. In the long run, generation of a library of prenylated chromenes will support Structure-Activity Relationship (SAR) studies for identification of novel compounds with prominent biological activity.

The seminar's motto: New Frontiers in the Chemistry of Natural Products has addressed numerous students and researchers acting in the fields of chemistry, biology, pharmacy, genetics, biophysics as well as specialists from the R\&D sector of companies with chemico-pharmaceutical profile. The general project overview was provided to the seminar audience.

Following presentations of project participants stressed the attention to the recent research directions of partner institutions. A special emphasize was placed on the use o modern analytical techniques for the structural elucidation of complex natural compounds, including those of marine origin. This issue was of special interest to Moldovan colleagues, due to the fact that modern analytical facilities, including a high-field NMR spectrometer became available in the Institute of Chemistry recently. Italian partners have shared their experience of world leaders in the area of marine chemistry.

Following multiple solicitations coming from seminar audience, made us provide in the current issue of the "Chemistry Journal of Moldova. General, Industrial and Ecological Chemistry" a condensed abstract of the seminar presentations. We do really hope that our joint event will give further impetus to our collaboration and will also motivate other researchers from Moldova to develop partnerships with European colleagues.

Dr. Margherita GAVAGNIN, CNR

Dr. Veaceslav KULCITKI, ASM

ASM-CNR joint project coordinators 